\title{
Use of Surveillance Outbreak Response Management and Analysis System for Human Monkeypox Outbreak, Nigeria, 2017-2019
}

\author{
Bernard C. Silenou, Daniel Tom-Aba, Olawunmi Adeoye, Chinedu C. Arinze, Ferdinand Oyiri, \\ Anthony K. Suleman, Adesola Yinka-Ogunleye, Juliane Dörrbecker, Chikwe Ihekweazu, Gérard Krause
}

In November 2017, the mobile digital Surveillance Outbreak Response Management and Analysis System was deployed in 30 districts in Nigeria in response to an outbreak of monkeypox. Adaptation and activation of the system took 14 days, and its use improved timeliness, completeness, and overall capacity of the response.

$\mathrm{H}$ uman monkeypox is a severe and rare smallpoxlike illness that occurs sporadically in remote villages in the tropical rain forest of West and Central Africa $(1,2)$. The causative agent, monkeypox virus, is transmitted by animal-to-human and human-tohuman contact $(3,4)$. In September 2017, an outbreak of monkeypox occurred in Nigeria after 40 years of no reported cases in the country. As of October 2017, local health departments in Nigeria had reported 89 cases and 294 contact persons $(5,6)$.

Early in the outbreak, the Nigeria Centre for Disease Control (NCDC) used a conventional surveillance system for the outbreak investigation. That system consisted of paper-based forms transferred manually to databases within the framework of the Integrated Disease Surveillance and Response System (7). As the outbreak expanded, NCDC faced challenges because of information delay and difficulties with updating and verifying case data, integrating laboratory tests, and managing contact tracing in the

Author affiliations: Helmholtz Centre for Infection Research,

Braunschweig, Germany (B.C. Silenou, D. Tom-Aba,

J. Dörrbecker, G. Krause); PhD Programme "Epidemiology,"

Braunschweig-Hannover, Germany (B.C. Silenou, D. Tom-Aba);

Nigeria Center for Disease Control, Abuja, Nigeria (O. Adeoye,

C.C. Arinze, F. Oyiri, A.K. Suleman, A. Yinka-Ogunleye,

C. Ihekweazu); German Center for Infection Research,

Braunschweig (G. Krause)

DOI: https://doi.org/10.3201/eid2602.191139 conventional system. In October 2017, NCDC decided to implement the Surveillance, Outbreak Response Management and Analysis System (SORMAS) on an ad hoc basis; an earlier prototype of this system had been successfully piloted in Nigeria in 2015 (8). SORMAS is an open-source mHealth (mobile health) system that organizes and facilitates infectious disease control and outbreak management procedures in addition to disease surveillance and epidemiologic analysis for all administrative levels of a public health system (9-11). SORMAS includes specific interfaces for 12 users (e.g., laboratorian, contact tracing officer, epidemiologist), disease-specific process modules for 12 epidemic-prone diseases, and a customizable process module for unforeseen emerging diseases; it adheres to the Integrated Disease Surveillance and Response System. Most users operate SORMAS on mobile digital devices (e.g., smartphone, tablet), bidirectionally synchronized with a central server via mobile telecommunication networks.

We compared SORMAS performance with that of the conventional surveillance system. Here we describe how we adapted and deployed SORMAS, discuss challenges encountered during implementation, and provide recommendations for deployment of similar mHealth tools.

\section{The Study}

In the second week of October 2017, we held a 2-day design thinking workshop with clinicians, epidemiologists, and virologists, in which all specific procedures for surveillance and response were defined in accordance with guidelines from the World Health Organization (12). Within 10 days, we translated the findings of the workshop into process models and programmed them into the existing SORMAS. A 2-day field test guided final programming revisions, 
Table 1. Qualitative comparison of attributes of SORMAS and the conventional surveillance system in response to monkeypox outbreak in Nigeria, November 2017-July 2019*

\begin{tabular}{|c|c|c|c|}
\hline Attribute & SORMAS & CS & Comments \\
\hline $\begin{array}{l}\text { Average time for data to arrive at NCDC from } \\
\text { LGAs }\end{array}$ & $2 \min$ & $2 \mathrm{~d}$ & $\begin{array}{l}\text { For the CS, the DSNOs sent the paper case forms by } \\
\text { post to NCDC, thus requiring longer time for case forms } \\
\text { to arrive at NCDC. }\end{array}$ \\
\hline $\begin{array}{l}\text { Average time to update data (sample results } \\
\text { from the laboratory, case classification, } \\
\text { outcome, contacts) per case }\end{array}$ & $5 \mathrm{~min}$ & $20 \mathrm{~min}$ & $\begin{array}{l}\text { Update in SORMAS requires searching for a case in the } \\
\text { case directory and directly updating the fields. For the } \\
\mathrm{CS} \text {, the database was Excel (https://www.microsoft.com), } \\
\text { and each type of case data was stored on a different } \\
\text { Excel sheet, thus increasing the time and complexity of } \\
\text { updating case data. }\end{array}$ \\
\hline $\begin{array}{l}\text { Workload to transfer cases from paper forms } \\
\text { to database at NCDC }\end{array}$ & Less & More & $\begin{array}{l}\text { With the CS, all case forms were entered in an Excel } \\
\text { database at NCDC; with SORMAS, } 90(38 \%) \text { of the } 240 \\
\text { cases were entered directly from the field by DSNOs. }\end{array}$ \\
\hline $\begin{array}{l}\text { Availability of dashboard and statistics module } \\
\text { to generate epidemiologic indicators for } \\
\text { disease surveillance (e.g., case classification } \\
\text { status, epidemic curve, laboratory test results, } \\
\text { fatalities, and map of spatial distribution of } \\
\text { cases and contact persons) }\end{array}$ & Yes & No & $\begin{array}{l}\text { SORMAS had a dashboard that displayed the needed } \\
\text { surveillance indicators; the CS did not. }\end{array}$ \\
\hline
\end{tabular}

which took another 2 days before the new module was released. In total, it took 14 days from initial decision to adapt and use SORMAS until its deployment.

In November 2017, we trained the laboratory officers and district surveillance notification officers (DSNOs) in 30 of the most affected local government areas of 8 federal states (Appendix, https:/ / wwwnc. cdc.gov/EID/article/26/2/19-1139-App1.pdf); each training session lasted 2 days. DSNOs used the mobile SORMAS version on mobile tablets to notify cases and conduct contact tracing; laboratories used either laptops or tablets to notify test results in SORMAS. We trained staff at the incident command center of the NCDC how to process and analyze data within SORMAS. The incident command center also transferred data into SORMAS received through the conventional system from local government areas not yet using SORMAS. The conventional system frequently involved recontacting DSNOs by phone to correct or update case reports. The dashboard and statistics module in SORMAS generated the epidemiologic indicators needed for weekly situation reports. We used the network package in R software for visualization and follow-up on chains of transmission (13). We conducted qualitative interviews with the NCDC incident managers of the monkeypox outbreak with regard to timeliness, usefulness, and workload of the conventional system compared with SORMAS. For quantitative evaluation, we used a set of core variables to compare the percentage of completeness in SORMAS versus that of the conventional system.

Yinka-Ogunleye et al. describe the epidemiologic characteristics of the outbreak in detail (14). From September 2017 through July 2019, including the period when SORMAS was not yet available, DSNOs reported 240 cases, either directly digitally in the field via SORMAS $(n=90)$ or via the conventional system $(n=150)$. Comparison of system attributes between SORMAS and the conventional system indicated equal or better performance of SORMAS for all attributes (Tables 1, 2). SORMAS continuously displayed the updated status of cases by case classification, epidemic curve, map of spatial distribution, contact persons, fatalities, and laboratory results, and it reported events in its dashboard within the incident command center (Figure 1). The dashboard also

Table 2. Quantitative comparison of attributes of SORMAS and the conventional surveillance system in response to monkeypox outbreak in Nigeria, November 2017-July 2019*

\begin{tabular}{lccc}
\hline Data availability for selected variables & SORMAS, \%† $\mathrm{n}=90$ & $\mathrm{CS}, \% \ddagger \mathrm{n}=150$ & $95 \% \mathrm{Cl}$ for difference \\
\hline Sex & 91 & 92 & $(-0.09$ to 0.07$)$ \\
Occupation & 84 & 57 & $(0.15$ to 0.39$)$ \\
Date of birth & 69 & 55 & $(0.00$ to 0.27$)$ \\
Onset date of symptoms & 89 & 85 & $(-0.06$ to 0.13$)$ \\
Body temperature & 53 & 3 & $(0.39$ to 0.62$)$
\end{tabular}

${ }^{*} 95 \% \mathrm{Cl}$ indicates difference in percentage of completeness determined by using 2-sample $\chi^{2}$ test. CS, conventional system; SORMAS, Surveillance Outbreak Response Management and Analysis System.

†Percentage of completeness for monkeypox cases notified directly in SORMAS by district surveillance officers in the field.

¥Percentage of completeness for monkeypox cases that arrived at the Nigeria Centre for Disease Control though the conventional system and were retrospectively registered in SORMAS. 


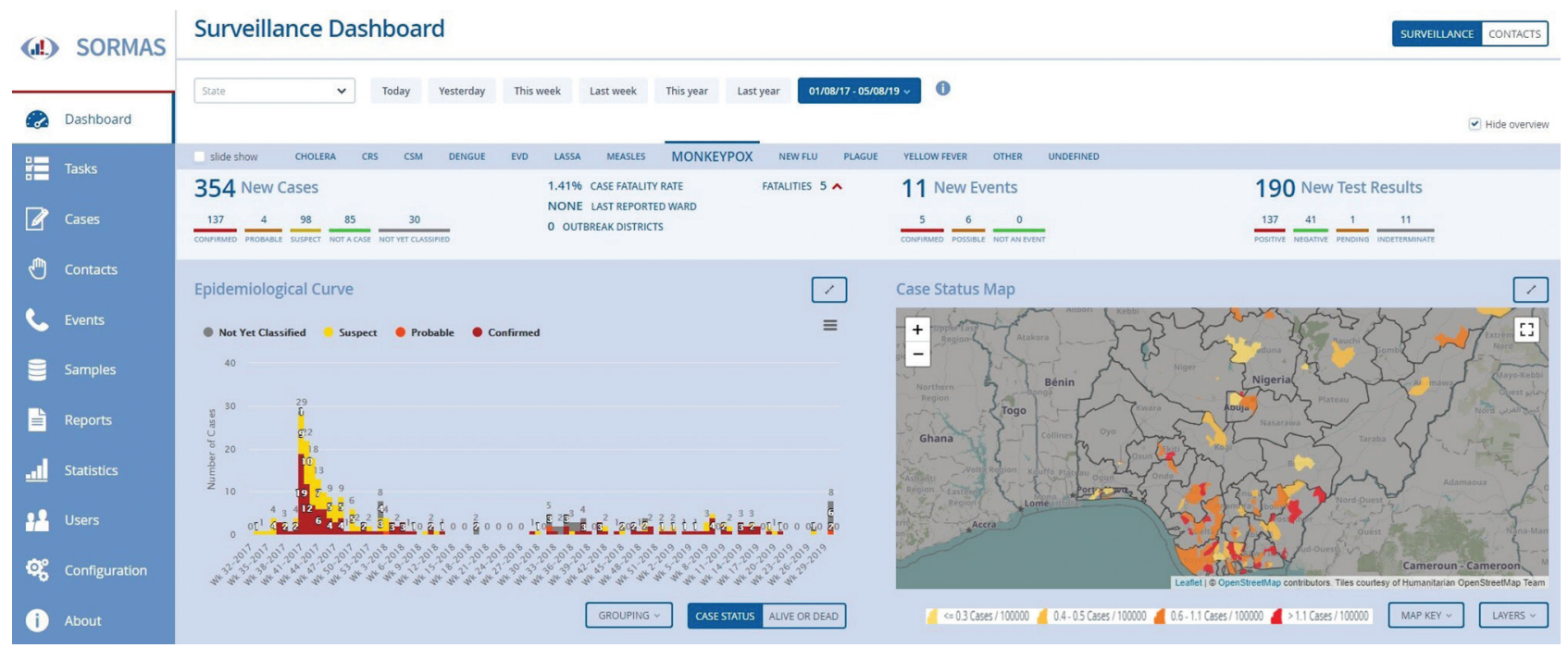

Figure 1. SORMAS dashboard showing monkeypox cases notified September 2017-July 2019 in Nigeria. The map shows the spatial spread of cases with local government area color by incidence proportion/100,000 population. The incidence proportion ranges from 0.1 (quartiles $0.3-0.7$ ) to 8.1 . During 2017, the number of cases by epidemic week increases gradually from week 32 to week 39 , sharply increases in week 40, and gradually declines until week 53. Exportation of graphs, tables, and other epidemic indicators was generated in the statistic module of SORMAS. SORMAS, Surveillance Outbreak Response Management and Analysis System.

included performance indicators on contact tracing and case follow-up. The network diagrams linking case-patients to contact persons demonstrate that, of 167 contact persons, $12(7 \%)$ converted to casepatients, of which $8(66 \%)$ emerged from 1 chain of transmission (Figure 2).

\section{Conclusions}

In this comparison, SORMAS clearly outperformed the conventional surveillance system. SORMAS accelerated visualization and analysis of case reports; expedited data updates and production of daily situation reports; and improved data completeness, timeliness, and several aspects of usefulness. The automated generation of chains of transmission enabled NCDC to assess overall transmissibility and effectiveness of contact tracing and helped with allocation of field staff during the outbreak.

The comparison of data completeness between SORMAS and the conventional system was limited by availability of data from the conventional system only after the incident command center had already executed data revisions and completions. Without this resource-intensive measure, the difference between SORMAS and the conventional system would have been more pronounced.

We also encountered challenges during the deployment phase. The ad hoc deployment of this new digital system in the midst of the outbreak allowed only 2 days of training for DSNOs to become acquainted with the tool. It also resulted in running 2 systems in parallel. Because the SORMAS concept integrates continuous surveillance and response management but has not yet been used routinely, its full potential could not come into play as the outbreak unrolled in this particular situation. Other challenges included the complaint of DSNOs not receiving compensation for transportation to execute follow-up visits for contact tracing, which could result in incomplete information about chains of transmission. This challenge, however, is not inherent to the conventional system or SORMAS, and SORMAS may have mitigated this challenge, given that it did produce chains of transmission that were not available by the conventional system.

Our evaluation was limited to selected attributes and based partly on quantitative analyses. Possibly the most convincing evidence for the added benefit of SORMAS was the ability of NCDC, while still responding to the monkeypox outbreak, to deploy SORMAS in 120 more local government areas of 6 federal states within 2 months. On the basis of the added value experienced through this measure, NCDC has set a goal to fully roll out SORMAS in all 774 local government areas of all 36 federal states plus the Federal Capital Territory in Nigeria by the end of 2021.

Overall, SORMAS has proven to be rapidly deployable and useful in response to multiple outbreaks, including an outbreak of an emerging disease such as monkeypox. For tools that integrate outbreak detection and response process management (such as SORMAS), we recommend their deployment 


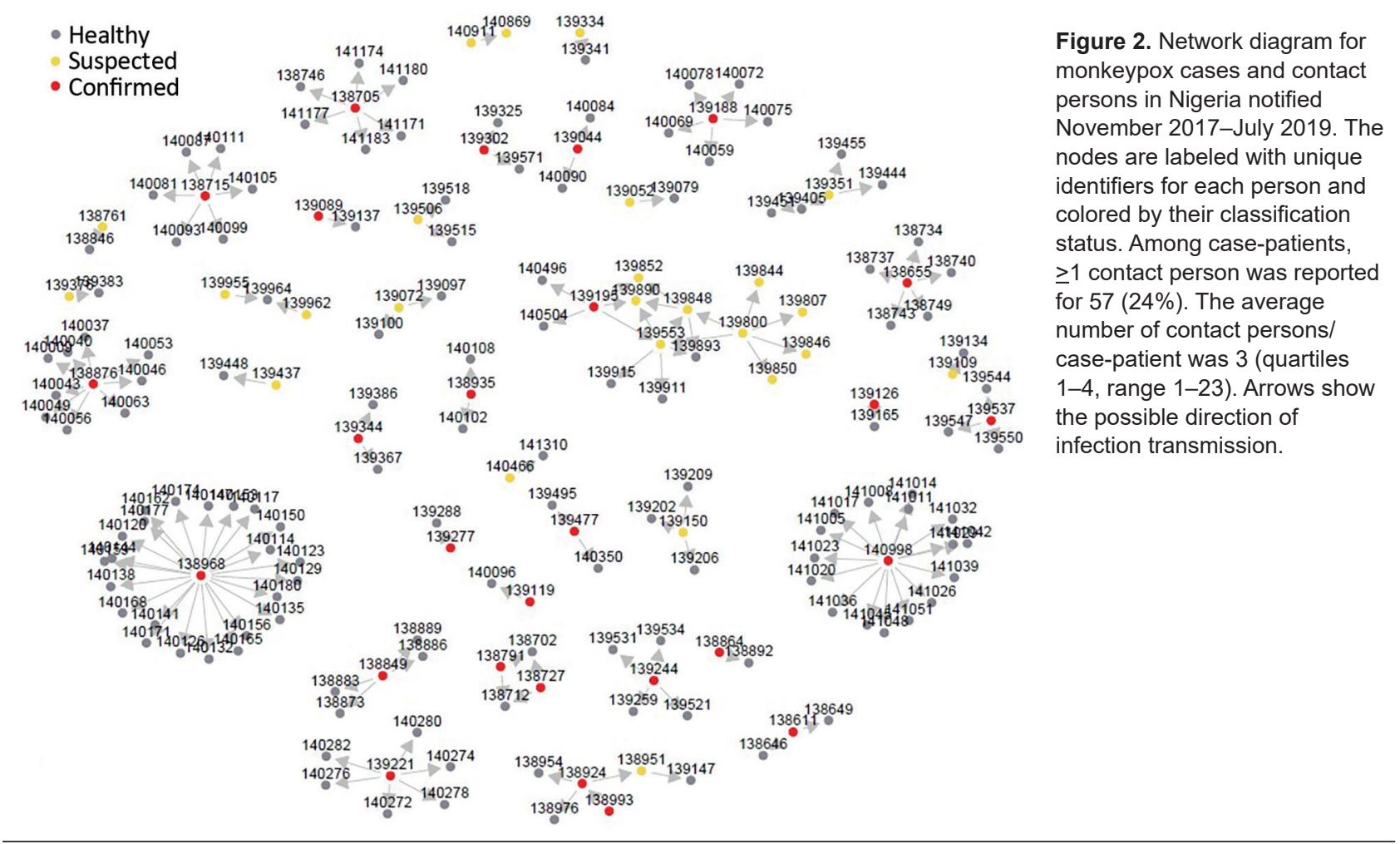

independently from any response to an acute public health emergency to optimize efficiency of resources for software adaptation, hardware infrastructure, and training. Such a proactive approach will improve not only outbreak response but also early detection of outbreaks, thus further enhancing sustainability.

\section{Acknowledgments}

We thank the field investigators and facilitators of the local and state health departments, NCDC, the African Field Epidemiology Network, and Symeda GmbH for contributing to the deployment of SORMAS in this outbreak and for providing valuable feedback for its improvement.

The mobilization of resources needed for the monkeypox-specific SORMAS deployment was made possible through financial support of the German Federal Ministry for Research and Education, the Gesellschaft für Internationale Zusammenarbeit, and the German Center for Infection Research.

\section{About the Author}

Mr. Silenou is working toward a PhD degree at the Helmholtz Centre for Infection Research and the Hannover Medical School in Germany. His primary research interests are investigation of the climatic factors that drive infectious disease outbreaks in Africa and research and development of digital surveillance systems.

\section{References}

1. Arita I, Jezek Z, Khodakevich L, Ruti K. Human monkeypox: a newly emerged orthopoxvirus zoonosis in the tropical rain forests of Africa. Am J Trop Med Hyg. 1985;34:781-9. https:/ / doi.org/10.4269/ ajtmh.1985.34.781

2. Breman JG, Kalisa-Ruti MV, Steniowski MV, Zanotto E, Gromyko AI, Arita I. Human monkeypox, 1970-79. Bull World Health Organ. 1980;58:165-82.

3. Ježek Z, Fenner F. Chapter VI. Epidemiology of human monkeypox. In: Ježek Z, Fenner F, editors. Human Monkeypox. Basel (Switzerland): Karger; 1988. p. 81-110.

4. Khodakevich L, Szczeniowski M, Manbu-ma-Disu, Ježek Z, Marennikova S, Nakano J, et al. The role of squirrels in sustaining monkeypox virus transmission. Trop Geogr Med. 1987;39:115-22.

5. Nigeria Centre for Disease Control. An update of monkeypox outbreak in Nigeria [cited 2019 May 6]. https://ncdc.gov.ng/diseases/ sitreps $/$ ?cat $=8 \&$ name $=$ An $\% 20$ Update $\% 20$ of $\% 20$ Monkeypox $\% 20$ Outbreak $\% 20$ in $\% 20$ Nigeria

6. Yinka-Ogunleye A, Aruna O, Ogoina D, Aworabhi N, Eteng W, Badaru S, et al. Reemergence of human monkeypox in Nigeria, 2017. Emerg Infect Dis. 2018;24:1149-51. https:// doi.org/10.3201/eid2406.180017

7. Centers for Disease Control and Prevention. Technical guidelines for integrated disease surveillance and response in the African region [cited 2019 May 6]. https:/ / stacks.cdc.gov/ view/cdc/12082

8. Adeoye O, Tom-Aba D, Ameh C, Ojo O, Ilori E, Gerado S, et al. Implementing Surveillance and Outbreak Response Management and Analysis System (SORMAS) for public health in West Africa-lessons learnt and future direction. Int J Trop Dis Health. 2017;22:1-17. https:/ / doi.org/10.9734/ IJTDH/2017/31584 
9. Fähnrich C, Denecke K, Adeoye OO, Benzler J, Claus H, Kirchner G, et al. Surveillance and Outbreak Response Management System (SORMAS) to support the control of the Ebola virus disease outbreak in West Africa. Euro Surveill. 2015;20:21071. https:/ / doi.org/10.2807/1560-7917. ES2015.20.12.21071

10. Tom-Aba D, Toikkanen SE, Glöckner S, Adeoye O, Mall S, Fähnrich C, et al. User evaluation indicates high quality of the Surveillance Outbreak Response Management and Analysis System (SORMAS) after field deployment in Nigeria in 2015 and 2018. Stud Health Technol Inform. 2018;253:233-7.

11. Tom-Aba D, Nguku PM, Arinze CC, Krause G. Assessing the concepts and designs of 58 mobile apps for the management of the 2014-2015 West Africa Ebola outbreak: systematic review. JMIR Public Health Surveill. 2018;4:e68. https://doi.org/10.2196/ publichealth.9015
12. World Health Organization. Technical Advisory Group on Human Monkeypox: report of a WHO meeting. Geneva, Switzerland; 1999 Jan 11-12 [cited 2019 May 4]. https:/ /apps.who.int/iris/handle/10665/65998

13. Butts CT. network: a package for managing relational data in $\mathrm{R}$. J Stat Softw. 2008;24:1-36. https:// doi.org/10.18637/jss.v024.i02

14. Yinka-Ogunleye A, Aruna O, Dalhat M, Ogoina D, McCollum A, Disu Y, et al.; CDC Monkeypox Outbreak Team. Outbreak of human monkeypox in Nigeria in 2017-18: a clinical and epidemiological report. Lancet Infect Dis. 2019;19:872-9. https://doi.org/10.1016/S1473-3099(19)30294-4

Address for correspondence: Gérard Krause, Epidemiology Department, Helmholtz Centre for Infection Research, Inhoffenstraße 7, 38124 Braunschweig, Germany; email: gerard.krause@helmholtz-hzi.de

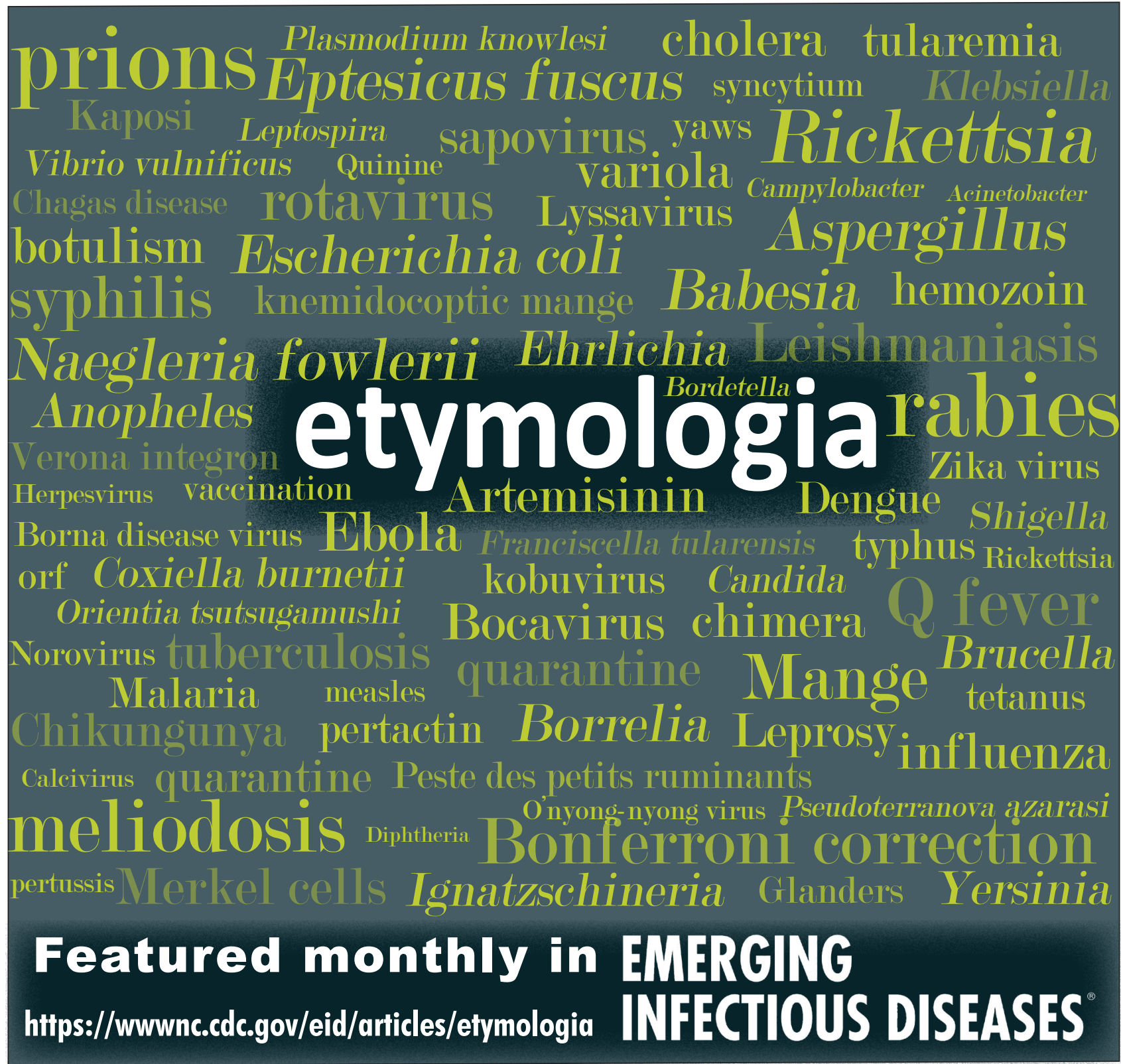

